\title{
CONOCIMIENTOS TRADicionales: ACERCAMIENTOS de Los Marcos Regulatorios de Propiedad INTELECTUAL ENTRE BRASIL Y MÉXICO
}

\author{
Salete Oro Boff* \\ Marta Carolina Giménez Pereira**
}

\begin{abstract}
1 Introducción. 2 Panorama de la medicina tradicional a fines del siglo pasado y comienzos del presente. 3 Conocimientos tradicionales y biodiversidad. 4 Protección jurídica sobre conocimientos tradicionales asociados. 4.1 Marco regulatorio de los conocimientos tradicionales en Brasil: la Constitución Federal de 1988, la Medida Provisoria 2186-16 de 2001 y la Ley 13123 del año 2015. Apuntes sobre el conocimiento tradicional asociado y el patrimonio genético. El caso de México en la figura de los conocimientos tradicionales. 5 Conclusión. Referencias.
\end{abstract}

\section{RESUMEN}

Los conocimientos tradicionales son concebidos como las informaciones transmitidas de generación en generación, de forma típicamente oral, compartidas por comunidades específicas y generadas en un contexto asociado con la cultura de grupo. Se insertan en un contexto de difícil delimitación frente al conocimiento científico. Esas implicancias dan margen a indagaciones sobre la apropiación de bienes considerados patrimonio de la humanidad, aunque fuese de forma simbólica, tal como la biodiversidad, para transformarlos en productos. De tal modo, el presente trabajo de investigación busca analizar la manera en que el nuevo marco regulatorio brasileño, la Ley 13.123/2015, prevé la repartición de beneficios en la comercialización de productos que se basan en el conocimiento tradicional. Se percibe que la nueva legislación flexibiliza el acceso a los conocimientos tradicionales y a la biodiversidad brasileña, con un indicativo gubernamental de expansión de la investigación y de la innovación y, por otro lado, con las inconformidades de las comunidades tradicionales, indicando conductas de carácter exploratorio y con un tinte tendenciosamente económico.

Palabras-clave: Conocimientos tradicionales. Medicina tradicional. Propiedad intelectual. Biodiversidad. Ley de Brasil 13.123/2015.

* Post-Doctora por la UFSC, Brasil. Doctora en Derecho por la UNISINOS, Brasil. Profesora del Programa de Posgrado en Derecho (Maestría) de la Universidad de Santa Cruz do Sul (UNISC). Línea de investigación: Políticas públicas de inclusión social. Grupo de investigación: Políticas públicas de inclusión social. Subgrupo: Políticas públicas para la innovación y la protección jurídica de la tecnología. Profesora e Investigadora de IMED - Faculdade Meridional, Brasil; profesora del Instituto Cenecista de Enseñanza Superior de Santo Angelo (IESA). E-mail: <salete.oro.boff@gmail.com>.

** Alumna de post-doctorado junto a UNAM - México, PPGD Capes - IMED. Doctora en Derecho / Instituto de Investigaciones Jurídicas, UNAM, México / PNPD CAPES no PPGDireito IMED. Linha de pesquisa Mecanismos de efetivação da democracia e da sustentabilidade. Grupo de pesquisa Direito, Novas Tecnologias e Desenvolvimento. E-mail: <magipe@hotmail.com>. 


\section{INTRODUCCIÓN}

Los conocimientos tradicionales, en adelante CT, son concebidos como las informaciones transmitidas de generación en generación, de forma típicamente oral, compartidas por comunidades específicas y generadas en un contexto asociado con la cultura de grupo. Tales conocimientos se insertan en un contexto de difícil delimitación frente al conocimiento científico. Los CT incluyen la medicina tradicional y también la artesanía, la música, la pintura y toda otra expresión artística producida en el seno de las comunidades indígenas.

Con tales consideraciones iniciales pretendemos identificar las distintas formas de protección jurídica de los CT relacionados con el término biodiversidad y corroborar las alteraciones que han sido efectuadas en el nuevo marco regulatorio brasilero, la Ley 13.123/2015. Pretendemos también analizar la previsión en la distribución de beneficios en la comercialización de los productos resultantes de los CT y realizar un estudio de las políticas de acceso al patrimonio genético brasileño.

Con tales propósitos, se estructura la concepción de los CT en un panorama legal internacional bastante exiguo a la fecha en lo que en específico a medicina tradicional se refiere, tal como en el siguiente apartado se describirá, pasando luego a una descripción relacionada con la biodiversidad para, seguidamente, presentar un punto de vista acerca del tratamiento dado a la protección jurídica conferida a esta figura propia de la Propiedad Intelectual, a saber contemplada en el ámbito internacional por el ADPIC o TRIPS (BOFF, 2006) por sus siglas en inglés y el Convenio sobre Diversidad Biológica (CDB) o Convención de Río y localmente, en Brasil, la Constitución Nacional de Brasil de 1988 (BRASIL, 1988), su Ley de Propiedad Intelectual y su Ley de Obtentor Vegetal. Finalmente esbozaremos las posibilidades que trae aparejada la reciente regulación brasileña de la mano de la Ley 13.123/2015, evidenciando la distribución de beneficios sobre el producido resultando del acceso a los CT.

La doctrina mexicana, por su parte, afirma que si bien se establecieron en el ámbito internacional nuevas formas de protección para las nuevas tecnologías desarrolladas en la modernidad, como la protección de bienes intangibles informáticos, programas de cómputo, bases de datos, señales de satélite y trazados de topografías de semiconductores (chips), patentamiento de microorganismos y organismos vivos o el sistema de protección de obtentores de vegetales bajo rasgos sui generis, no parece imposible impulsar de igual manera sistemas originales para proteger el CT, permitiendo incluso su comercialización. Para ello, se necesita un rápido avance en acuerdos sobre respuestas concretas a muchos de los problemas subsistentes al respecto, para lo cual el punto de partida es comenzar por establecer qué tipo de conocimientos se pretende proteger (MIRANDA, 2011, p. 195) ya que estos mismos aspectos de la Propiedad Intelectual al ser per se sui generis necesitan de igual modo una protección diferenciada.

Estudiosos en México, países con una enorme diversidad cultural, biológica y socioeconómica, se encarga de subdividir lo que denomina Propiedad Intelectual Sui Generis en 
tres ramas: la flora y fauna originaria; los conocimientos tradicionales (MIRANDA, 2011), y los derechos del agricultor. Sobre los CT es sobre lo que nos ocuparemos más adelante.

La protección jurídica que el CT encuentra en México tiene base en el artículo 2 de su Constitución Nacional. Dentro de su marco legal se menciona que este país ha ratificado el CDB en el año 1992, así como en 1990 el Convenio 169 de la Organización Internacional del Trabajo (OIT, 1989) sobre Pueblos Indígenas y Tribales, el cual ha sido ratificado en su mayoría por países iberoamericanos. ${ }^{1}$ Otras normativas son: la Ley General del Equilibrio Ecológico y la Protección al Ambiente (LGEEPA) (MÉXICO, 1988) de 1988, cuya última reforma data de 2012; la Ley General de Vida Silvestre (LGVS) de 2000 (MÉXICO, 2000), cuya última reforma se dio el pasado año 2016; la Ley General de Desarrollo Forestal Sustentable (LGDFS) del año 2003 (MÉXICO, 2003), reformada en 2012; y la Ley General de Pesca y Acuacultura Sustentables (LGPAS) de 2007 (MÉXICO, 2007), reformada en 2015, todas las cuales mencionan la figura del conocimiento tradicional como referencia a los derechos que poseen los indígenas pero sin constituir ninguna de ellas una legislación específica de esta figura de la Propiedad Intelectual.

\section{PANORAMA DE LA MEDICINA TRADICIONAL A FINES DEL SIGLO PASADO Y COMIENZOS DEL PRESENTE}

La ley de Taiwán (Taipei, República de China) se refiere en su artículo 19 a la invención como una elevada creación de concepto(s) técnicos(s) para los cuales se utilizan las leyes naturales. De hecho, es una de las pocas legislaciones que definen el concepto abarcando la naturaleza.

Este país, al igual que otros que cuentan con extraordinaria y milenaria tradición como Brasil, México, India, Australia, Paraguay, Perú, Bolivia, Nueva Zelanda, se encuentran abocados a la obtención de una protección integral en materia de medicina tradicional, la cual se encuentra falta de mecanismos de defensa adecuados no sólo a la protección de las invenciones en sí sino también al respeto de los mismos derechos humanos de sus poblaciones, máxime teniendo presente que en la globalización que enfrentan la participación de sus minorías es casi nula y toca más bien al Estado velar por sus intereses.

En el plano internacional, es la Organización Mundial de la Salud (OMS) la institución que cuenta con uno de los más amplios programas que contempla la materia. La OMS define a la medicina tradicional como un conjunto de prácticas, conocimientos y creencias en materia de salud "que se basan en la utilización de medicinas que tienen su origen en plantas, animales y minerales, así como terapias espirituales, técnicas manuales y ejercicios aplicados en forma individual o en combinación para tratar, diagnosticar y prevenir enfermedades o mantener el bienestar." (WORLD HEALTH ORGANISATION, 2010, online).

El programa que desarrolla este organismo busca integrar la medicina tradicional en los diferentes sistemas nacionales para el cuidado de la salud, así como establecer estándares 
internacionales para la investigación de la medicina tradicional y actuar como una oficina de enlace en el intercambio de información. Así, a principios de este siglo, de este organismo han surgido las "Estrategias 2002-2005 en medicina tradicional" tras una serie de negociaciones sobre la materia entre los países miembros y las distintas áreas involucradas. Específicamente se busca reforzar los objetivos del programa mencionados así como promover en los países miembros la regulación de una rama tan importante del CT como lo es la medicina herbolaria, garantizar el uso y desarrollo sustentable de las plantas medicinales y proteger y preservar los conocimientos en medicina tradicional de las comunidades indígenas.

Asimismo, existe desde el año 2000 un Comité Intergubernamental de la Organización Mundial de la Propiedad Intelectual (OMPI) en Recursos Genéticos, Conocimiento Tradicional y Folklore, en la que participan activamente las Organizaciones no Gubernamentales además de los países miembros, todos a un mismo nivel de debate, lo cual hace que las partes involucradas se encuentren en igualdad de condición al menos a la hora de discutir, pues es bien sabido que el sector representado por los indígenas es siempre el más débil y resulta finalmente más desprotegido en la toma de decisiones (PÉREZ, 2006).

La forma jurídica que se adopta para la obtención de beneficios en la explotación de la medicina tradicional y otros usos de la biodiversidad entre las comunidades indígenas y las empresas multinacionales es por excelencia el contrato. Se ahondará al respecto de la biodiversidad más adelante en este trabajo.

Enfatizamos que los CT no sólo incluyen la medicina sino también la artesanía, la música, la pintura y toda otra expresión artística producida en el seno de las comunidades indígenas. El objetivo normativo que tienen en común estas diversas manifestaciones es el de lograr en un futuro cercano la protección adecuada del intelecto y que la compensación económica suficiente a estas comunidades sean una realidad, más aún porque casi siempre representan el sector más indefenso y vulnerable de la población en todos los ámbitos del derecho. Si bien se cuenta ya con una interesante gama legislativa sobre el tema, aún queda mucho por regular para salvaguardar los intereses de este producto del intelecto y sostenemos que lo existente sigue siendo exiguo.

Entre las legislaciones internacionales se destaca el Convenio sobre Diversidad Biológica (CDB) o Convención de Río, que se abrió a firma en el marco de la llamada Cumbre de la Tierra, celebrada en Río de Janeiro, Brasil, el 5 de junio de 1992, entrando en vigor el 29 de diciembre de 1993. Este es el tratado internacional por excelencia en la materia. Establece claramente las formas y mecanismos de protección a los grupos indígenas y a sus creaciones, sobre todo en cuanto a los beneficios que se derivan a fin de ser distribuidos en forma justa, preservando el fin principal que es la conservación y el uso adecuado de la biodiversidad.

El CDB trata de prever, prevenir y atajar en su fuente las causas de reducción o pérdida significativa de la diversidad biológica, debido a su valor intrínseco y a los valores de sus componentes medioambientales, genéticos, sociales, económicos, científicos, educativos, culturales, recreativos y estéticos. El Convenio trata igualmente de promover la cooperación entre los Estados miembros y las organizaciones intergubernamentales. 
En efecto, el debate sobre los conocimientos tradicionales se vinculó desde un inicio con la biopiratería y el acceso a los recursos genéticos, lográndose un primer reconocimiento de los derechos de las naciones en el derecho internacional, en el art. $3^{\circ}$ y $8^{\circ}$ del CDB. Ha sido uno de los temas más difíciles de reglamentar por las diferencias conceptuales, debidas en gran medida a las peculiaridades ambientales y sociales de los países involucrados. A ello se agrega una gran disputa internacional por los recursos genéticos que se ha desatado en las últimas décadas em los países industrializados. En razón de ello, también ha sido difícil desarrollar instrumentos internacionales y nacionales que permitan a las comunidades locales e indígenas y a los Estados Partes del CDB, ejercer este derecho. El mayor avance hasta la fecha en el ámbito internacional ha sido el Protocolo de Nagoya sobre Acceso a los Recursos Genéticos y Participación Justa y Equitativa en los Beneficios que se Deriven de su Utilización al Convenio sobre la Diversidad Biológica, o "Protocolo de Nagoya" firmado en Nagoya, Japón, el 29 de octubre de 2010 y que reglamenta detalladamente las disposiciones del CDB sobre acceso a los conocimientos tradicionales y a los recursos genéticos (MIRANDA; PICHARDO, 2017).

El CDB cuenta con una novedosa disposición en el mencionado artículo 3 y en el artículo 15.1 al establecer que son los Estados quienes tienen el derecho soberano de explotar sus propios recursos en aplicación de su propia política ambiental. Anteriormente, se sostenía la tendencia de que dichos recursos eran patrimonio de la humanidad. Para mayor aclaración citamos lo que a la letra la Convención de Río en el referido artículo 3 expresa:

Principio. De conformidad con la Carta de las Naciones Unidas y con los principios del derecho internacional, los Estados tienen el derecho soberano de explotar sus propios recursos en aplicación de su propia política ambiental y la obligación de asegurar que las actividades que se lleven a cabo dentro de su jurisdicción o bajo su control no perjudiquen al medio de otros Estados o de zonas situadas fuera de toda jurisdicción nacional (ORGANIZACIÓN DE LAS NACIONES UNIDAS, 1993, online).

El artículo 15 a su vez reza: "Acceso a los recursos genéticos. 1. En reconocimiento de los derechos soberanos de los Estados sobre sus recursos naturales, la facultad de regular el acceso a los recursos genéticos incumbe a los gobiernos nacionales y está sometida a la legislación nacional.”(ORGANIZACIÓN DE LAS NACIONES UNIDAS, 1993, online).

Por su parte destacamos su artículo 8 que expresa en su inciso J que los Estados deberán prestar especial cuidado en la elaboración de sus legislaciones respectivas y que deberá respetarse las innovaciones de las comunidades indígenas, la cual deberá fomentar la compensación efectiva. A la letra, el artículo 8 de la Convención de Río establece:

Conservación in situ. Cada Parte Contratante, en la medida de lo posible y según proceda : J. Con arreglo a su legislación nacional, respetará, preservará y mantendrá los conocimientos, las innovaciones y las prácticas de las comunidades indígenas y locales que entrañen estilos tradicionales de vida pertinentes para la conservación y la utilización sostenible de la diversidad biológica y promoverá su aplicación más amplia, con la aprobación y la participación de 
quienes posean esos conocimientos, innovaciones y prácticas, y fomentará que los beneficios derivados de la utilización de esos conocimientos, innovaciones y prácticas se compartan equitativamente. (ORGANIZACIÓN DE LAS NACIONES UNIDAS, 1993, online).

A objeto de cumplir con el objetivo establecido en este artículo, se reúne periódicamente un grupo de trabajo ad hoc, intentando darle un papel más activo a las comunidades indígenas, al tiempo de solicitar a los gobiernos que hagan suyas las decisiones tomadas e iniciar su estudio a fondo con el objeto de incorporarlas paulatinamente en sus respectivas legislaciones (PÉREZ, 2006, p. 25-26).

Otro instrumento internacional importante a destacar, Convenio 169 de la Organización Internacional del Trabajo (OIT) sobre Pueblos Indígenas y Tribales, el cual menciona en sus artículos 15, primer apartado: "Los derechos de los pueblos interesados a los recursos naturales existentes en sus tierras deberán protegerse especialmente. Estos derechos comprenden el derecho de esos pueblos a participar en la utilización, administración y conservación de dichos recursos" (ORGANIZACIÓN INTERNACIONAL DEL TRABAJO, 1989, online) y en su segundo apartado explica con claridad que

[...] en caso de que pertenezca al Estado la propiedad de los minerales o de los recursos del subsuelo, o tenga derechos sobre otros recursos existentes en las tierras, los gobiernos deberán establecer o mantener procedimientos con miras a consultar a los pueblos interesados, a fin de determinar si los intereses de esos pueblos serían perjudicados, y en qué medida, antes de emprender o autorizar cualquier programa de prospección o explotación de los recursos existentes en sus tierras agregando que los pueblos tienen la obligación de participar siempre que puedan en los beneficios resultantes así como el derecho de percibir una indemnización equitativa por cualquier daño sufrido como resultado de tales actividades. (ORGANIZACIÓN INTERNACIONAL DEL TRABAJO, 1989, online).

Claramente se percibe que conforme a esta legislación no es facultativa de los pueblos participar de los programas que involucren sus propios recursos, sino más bien una obligación de los mismos.

\section{CONOCIMIENTOS TRADICIONALES Y BIODIVERSIDAD}

Contextualizando, el conocimiento es lo que está sujeto al espacio cultural y a sus actores, como resultado de una diversidad de iniciativas creativas e innovadoras que comulgan a lo largo de los tiempos y se plasman en forma de usos, creencias, invenciones, descubrimientos y perfeccionamientos de técnicas y productos. Por lo tanto, es el resultado de una comunión de conocimiento empírico con conocimiento científico (comprobados) (GORZ, 2005, p. 10;32-33).

Por la denominación "conocimientos tradicionales" (CT) se entiende las informaciones y las prácticas de comunidades (indígenas, de cimarrones, ribereñas u otras que viven en estrecha relación con el ambiente), que puedan transformarse en valor, asociadas al patrimonio genético. Por ejemplo, es el conocimiento acerca de las virtudes curativas de determinado 
vegetal, propiedades que se transmiten entre las generaciones de forma mayormente oral. Se presentan logradamente por la Organización Mundial de la Propiedad Industrial (OMPI) como aquellos conocimientos resultantes de una práctica diaria, "creados cada día y desarrollados como una respuesta de personas y comunidades a los desafíos puestos por su medio social y físico." (ADIERS, 2002, p. 59).

Es una práctica común utilizar los CT como punto de partida para encaminar investigaciones hacia el estudio del potencial tanto farmacológico, como se vio en el apartado anterior respecto a la medicina tradicional, como también comestible de determinada planta. Se recogen los recursos biológicos considerando los indicadores visibles de utilización del uso común por los grupos y de ahí se pasa al estudio de los mismos. Al asociar el CT al científico, se da un paso enorme en pro del éxito de las investigaciones y tal práctica puede desembocar en el patentamiento de los productos y procedimientos (BARBOSA, 1998, p. 69).

Cuando existe apropiación foránea del conocimiento sin consentimiento de los pueblos tradicionales es cuando ocurre la biopiratería, resultando en una privatización del conocimiento colectivo. Las comunidades locales proveen el material e inclusive las informaciones respecto a las cualidades alimenticias o curativas. Los materiales son encaminados a laboratorios especializados en países desarrollados, donde son aislados e identificados los genes para la posterior solicitud de patentes. ${ }^{2}$

Inmersa en una preocupación con la preservación de la diversidad biológica en razón de la creciente destrucción del ambiente natural y de la apropiación del conocimiento tradicional, la CDB, también llamada ECO92, en su artículo 1 destaca como objetivo la

[...] conservación de la diversidad biológica, la utilización sostenible de sus componentes y la participación justa y equitativa en los beneficios que se deriven de la utilización de los recursos genéticos, mediante, entre otras cosas, un acceso adecuado a esos recursos y una transferencia apropiada de las tecnologías pertinentes, teniendo en cuenta todos los derechos sobre esos recursos y a esas tecnologías, así como mediante una financiación apropiada (ORGANIZACIÓN DE LAS NACIONES UNIDAS, 1993, online). ${ }^{3}$

La Convención, nótese, se preocupa en mantener y conservar los conocimientos y las prácticas tradicionales de las comunidades locales por medio (y allí enumera sólo enunciativamente) del uso sostenible de los recursos naturales, de la mano con la preservación del ecosistema de que se trate, pues la "biodiversidad siempre fue un recurso local comunitario" (ADIERS, 2002, p. 56), que combina derechos y una responsabilidad entre sus usuarios, "un sentido de coproducción con la naturaleza y de dádiva entre los miembros de la comunidad."(SHIVA, 2001, p. 92-93).

Recordemos que, per se, el ser humano es contextual, temporal - limitado por su misma temporalidad física- y geográfico - al formar parte de una determinada nación ${ }^{4}$ la cual sí es en cambio de tendencia permanente y estable-, o sea, el hombre es un ser ambiental inserto e inmerso en el medio ambiente que le caracteriza y hacia el cual se refleja.

En México se cita el caso del proceso para generar semilla y maíz para el consumo en 
la localidad de Yaxcabá, Yucatán. Existen cuatro procesos que intervienen en la gestión del conocimiento tradicional para el cultivo del maíz en esa localidad: selección, mejoramiento, conservación y diversidad morfológica. Así, el proceso de selección incluye la elección de la semilla y el lugar donde se realizará la próxima siembra; el mejoramiento se materializa por medio de la cruza y acriollamiento entre variedades; la conservación se hace tal tanto en los solares como en las milpas; y la diversidad morfológica aparece en las características fenotípicas de diferentes productos. Todos estos procesos y las variables o elementos que entran en juego, así como los productos y subproductos que se obtienen de la sistematización y ejecución de procesos, y las semillas que responden a condiciones climatológicas propias de esa región, caracteriza la agricultura maya que cultiva variedades locales según principios de cultura y necesidades de alimentación y se puede afirmar que la selección ciclo tras ciclo de la semilla influye en el mejoramiento, pues el campesino sabe qué características elegir de la semilla para poder aprovecharlas mejor. Por ende, "el mejoramiento contribuye a la generación de la variabilidad morfológica del maíz que poseen los denominados milperos de Yaxcabá" y responden a un objetivo tan específico como la venta de un maíz de mayor aceptación en el mercado y obedece a todo un proceso de modificación de variedades a través de un proceso de acriollamiento indispensable en el lugar específico donde se siembre, por sus condiciones propias sin la cuales no se obtendría una cosecha aceptable de maíz que satisfaga el gusto del consumidor. (ARGÜELLO; RAMOS; CADENA, 2008, online).

\section{PROTECCIÓN JURÍDICA SOBRE CONOCIMIENTOS TRADICIONALES ASOCIADOS}

Considerando la proximidad y las derivaciones entre el CT y el científico al utilizar herramientas de la biodiversidad, corresponde al derecho regular y garantizar reglas que delimiten los derechos inherentes a las comunidades tradicionales. Los temas se relacionan directamente con el Derecho de Propiedad, garantizado por la Constitución Federal, el cual abarca el Derecho a la Propiedad Privada, condicionado a su función social. Sobre el particular se refleja una función primordial del Derecho de Propiedad Intelectual que comprende el conjunto de derechos que gozan los autores de obras intelectuales sobre sus creaciones que emanan del espíritu, facultando a sus titulares derechos económicos que dictan una forma de comercialización, circulación, utilización y producción de los bienes intelectuales y de los productos y servicios que incorporan tales creaciones. La Propiedad Intelectual ${ }^{5} \mathrm{comprende}$ el Derecho de Autor y los Derechos Conexos o vecinos, la Propiedad Industrial así como otras formas denominadas sui generis. El Derecho de Autor comprende las creaciones de lo imaginario humano. En el campo de la Propiedad Industrial ${ }^{6}$ están las patentes, las marcas, los diseños y modelos industriales, las indicaciones geográficas así como la competencia desleal. Es el producto de la invención y de la expresión creativa así como la protección pública que se concede (SHERWOOD, 1992, p. 22). Entre las formas sui generis se incluyen 
la protección de software (protección concedida por el Derecho de Autor así como por el registro de patente), los obtentores vegetales, topografía de circuitos integrados y, por fin, los conocimientos tradicionales.

Se percibe de ese modo la importancia de la Propiedad Intelectual en el contexto del desarrollo tecnológico y en la medida del valor que agrega al conocimiento. En muchas situaciones, la protección del Derecho de Propiedad Intelectual privilegia los derechos de los descubridores, incluso cuando tales revelaciones se amparan en los CT imprimidos por los pueblos con el pasar de los tiempos.

Un hecho que llevó al incremento del reconocimiento de los productos tradicionales fue la concesión por los Estados Unidos de privilegios a organismos vivos per se cuando son "obtenidos en complicadas investigaciones genéticas." En razón de eso, "gigantes empresariales financian expediciones por todo el hemisferio sur en busca de huellas genéticas raras y originales que puedan tener algún valor comercial.” El gran interés está en el valor que las nuevas drogas derivadas de plantas, prescriptas en nuestros días y que eran utilizadas en la medicina indígena. "El Curare, por ejemplo, que es un importante anestésico quirúrgico y relajante muscular, se deriva de extractos vegetales utilizados por los indios del Amazonas para paralizar la caza." (RIFKIN, 1999, p. 52). Otro caso es el patentamiento de procesos de utilización del neem, árbol nativo de la India (RIFKIN, 1999, p. 52-53). Esa patente fue considerada como una tentativa empresarial de patentar conocimientos indígenas y recursos biológicos nativos. El aislamiento del componente del neem, la azadiractina, por A.W.R. Grace, recibió varias patentes para los métodos y procesos utilizados para la producción del extracto mencionado. Pero los privilegios fueron reclamados por los científicos hindús, comprobando que los métodos y los procesos empleados ya eran utilizados hace siglos y que, en razón de tal hecho, las informaciones deberían "ser libres y abiertamente compartidas."

\subsection{MARCO REGULATORIO DE LOS CONOCIMIENTOS TRADICIONALES EN BRASIL: LA CONSTITUCIÓN FEDERAL DE 1988, LA MEDIDA PROVISORIA 2186-16 DE 2001 Y LA LEY 13123 DEL AÑO 2015. APUNTES SOBRE EL CONOCI- MIENTO TRADICIONAL ASOCIADO Y EL PATRIMONIO GENÉTICO. EL CASO DE MÉXICO EN LA FIGURA DE LOS CONOCIMIENTOS TRADICIONALES}

Los CT asociados a los recursos, cuya naturaleza definiremos más adelante en este mismo apartado, no están incluidos dentro de las figuras de las patentes en la Ley de Propiedad Industrial 9279 de 1996 (MÉXICO, 1991). Del mismo modo se presenta imposible proteger los conocimientos tradicionales por medio del Derecho de Autor, en la Ley 9610 de 1998, que tiene como requisito la originalidad de la obra. Igualmente, la Ley de Obtentor Vegetal 9456 de 1997, que protege las variedades vegetales, se refiere a la planta como un todo, considerando el conjunto de sus características. La protección de la figura de obten- 
tor vegetal no sigue rígidamente los requisitos propios de las patentes de invención, siendo los requisitos para el registro del vegetal las características de distintividad, homogeneidad y estabilidad. Sin embargo, a pesar de estar detallada la materia, no hay una referencia en cuanto a la protección de los CT asociados.

La Medida Provisoria (MP) 2186-16 de 2001, da inicio a una regulación específica en Brasil respecto a los CT asociados, relacionándolos al patrimonio genético existente y la plataforma continental y la zona económica exclusiva. La MP creó el Consejo de Gestión de Patrimonio Genético (CGEN) vinculado al Ministerio del Medio Ambiente y estableció el pago de regalías sólo para la transferencia de tecnologías sujetas a patente.

Además de los derechos de Propiedad Intelectual, se destacan a modo ejemplificativo los Derechos de Autor que corresponden a los indígenas y que se hallan contemplados en el texto constitucional brasileño de 1988, específicamente en su artículo 231, en el cual se reconoce la organización social, las costumbres, la lengua, las creencias, las tradiciones indígenas y el carácter colectivo de las mismas y garantiza derechos sobre sus bienes materiales e inmateriales. La Constitución reconoce las manifestaciones culturales indígenas que constituyen su patrimonio cultural. La ley infra-constitucional federal y estatal se ocupa de la temática, tal como resulta del Estatuto del Indio, Ley 6001/73, que asegura genéricamente el respeto al patrimonio cultural de las comunidades indígenas y castiga algunas acciones que violan el derecho a la propia imagen y expresiones de su cultura (INSTITUTO SOCIOAMBIENTAL, 2017). Aún así y a pesar del amparo de la ley, los derechos inmateriales que constituyen la expresión de sus culturas, tales como los cantos, los dibujos, las pinturas y los mitos, a menudo han sido "utilizados de manera indebida y sin que se les haya facultado el acceso a instrumentos eficaces de protección de sus derechos. El acoso y los atropellos en cuanto al uso de la imagen y de los bienes culturales indígenas crecen en cantidad y en complejidad [...]."(INSTITUTO SOCIOAMBIENTAL, 2017, online).

En 2015 fue editada la Ley 13123 (BRASIL, 2015) ${ }^{8}$ que reglamentó el inciso II del $\S$ 1ํ y el $\S$ 4 del artículo 225 de la Constitución y el artículo 1, el numeral J del artículo 8, el numeral c del artículo 10, así como el artículo 15 y los $\$ \S 3$ y 4 del artículo 16 de la CDB. El texto legal dispone sobre el acceso al patrimonio genético, sobre la protección y el acceso al CT asociado y sobre la repartición de beneficios para la conservación y el uso sostenible de la biodiversidad, además de derogar la MP 2186-16 de 2001.

Las nuevas reglas establecen directrices para el acceso al patrimonio genético y al CT asociado para fines de investigación y desarrollo tecnológico, así como la distribución de los beneficios que resulten de la explotación económica de producto o material reproductivo desarrollado a partir de esos accesos.

En los artículos 1 y 2 de la ley se enuncian varias definiciones sobre el tema. En principio se consideran que integran el patrimonio genético las especies, las variedades tradicionales, las razas adaptadas y criollas, las especies introducidas en el territorio nacional por la acción 
humana y los microorganismos aislados. De igual modo, se considera que conforman tal patrimonio genético la investigación o desarrollo tecnológico realizado sobre muestra de patrimonio genético y, como parte del CT asociado, la investigación o desarrollo tecnológico realizado sobre ese $\mathrm{CT}$ asociado cuando viabiliza el acceso al patrimonio genético.

Definiendo, el "conocimiento tradicional asociado" es la información o la práctica del pueblo indígena, comunidad tradicional o agricultor tradicional sobre las propiedades o usos, directos o indirectos, asociada al patrimonio genético, mientras que el "conocimiento tradicional de origen" no identificable es aquel en el que no hay posibilidad de vincular a su origen a un pueblo indígena, una comunidad tradicional o un agricultor tradicional. Una comunidad tradicional es un grupo culturalmente diferenciado que se reconoce como tal, que posee una forma propia de organización social y que ocupa y usa territorios y recursos naturales como condición para su reproducción cultural, social, religiosa, ancestral y económica, utilizando conocimientos, innovaciones y prácticas generadas y transmitidas por la tradición. El usuario de ese conocimiento puede ser persona física o jurídica que realiza el acceso o que explora económicamente el producto acabado o el material reproductivo oriundo de ese acceso al patrimonio genético o al CT asociado.

El órgano responsable en implementar esa nueva legislación será el Consejo de Gestión del Patrimonio Genético (CGEN) que tendrá como tarea evaluar, aprobar o reprobar propuestas públicas y privadas de investigación y uso económico del patrimonio genético y del respectivo CT asociado. Se conformará por un sesenta por ciento de representantes de órganos y entidades de la Administración Federal y por un 40\% de representantes de la sociedad civil, garantizando una paridad entre el sector empresarial, el académico y el indígena, así como las comunidades locales y los agricultores tradicionales. Una de sus funciones también es establecer directrices para aplicar los recursos destinados para el Fondo Nacional para la Distribución de Beneficios, o FNRB por sus siglas en portugués, así como promover debates y consultas públicas sobre este mismo tema.

La ley hace distinción entre el CT identificable, que es aquel que se atribuye a un determinado grupo, y no identificable, que es el que es difuso. En este caso, el valor del porcentaje va para un fondo común (tal es el caso del té quebra pedra). Cuando se trata del acceso al CT asociado de origen identificable se tiene como condición la obtención del consentimiento previo informado. La comprobación del mismo podrá ocurrir a criterio de la población indígena, de la comunidad tradicional o del agricultor tradicional, con la firma del acuerdo pertinente o por medio de registro audiovisual del consentimiento o según parecer del órgano oficial competente o, incluso, por la adhesión según formas previstas en el protocolo comunitario. Queda palpable la duda de qué se entiende por "parecer del órgano oficial competente" en el sentido de que este tipo de comprobación podría tornarse bastante subjetivo. Por otra parte, hay que destacar que cuando el acceso se refiere al CT asociado de origen no identificable o difuso, no existe la necesidad de consentimiento previo informado.

Subrayamos que las comunidades tradicionales tienen derecho: al reconocimiento de su contribución para el desarrollo y conservación del patrimonio genético en cualquier forma 
de publicación, utilización, exploración y divulgación; a tener indicado el origen del acceso al CT asociado en todas las publicaciones, utilizaciones, exploraciones y divulgaciones; a percibir beneficios por la exploración económica por terceros, directa o indirectamente, del CT asociado en los términos de la legislación en comento; a participar del proceso de toma de decisión sobre asuntos relacionado al acceso al CT asociado y a la repartición de beneficios como consecuencia de ese acceso y en la forma prevista en la regulación; a usar o vender libremente productos que contengan patrimonio genético o CT asociado una vez observadas las disposiciones de la Ley de Obtentor Vegetal; y a conservar, manejar, guardar, producir, intercambiar, desarrollar y mejorar el material reproductivo que contenga patrimonio genético o CT asociado, conforme se prevé en el artículo 10.

En cuanto a la distribución de beneficios, la nueva regulación prevé la distribución de beneficios de la exploración económica del producto acabado o de material reproductivo desarrollado a partir del acceso al CT asociado o al patrimonio genético realizado sobre muestra de especie vegetal y animal, inclusive la domesticada, encontrada en condiciones in situ en el territorio nacional, en la plataforma continental, en el mar territorial y en la zona económica exclusiva. Incluye asimismo en la división de beneficios la especie vegetal, animal, microbiana mantenida en condiciones ex situ, siempre que haya sido recogida en condiciones in situ en el territorio nacional, en la plataforma continental, en el mar territorial y en la zona económica exclusiva la variedad tradicional, sea local o criolla. Incluso establece la división de beneficios sobre el acceso a la raza localmente adaptada o criolla, a la especie introducida en el territorio nacional por la acción del hombre que forme una población espontánea y que haya adquirido una característica distintiva propia en el país así como el acceso al microorganismo que haya sido aislado a partir de substrato recogido en el territorio nacional, mar territorial, plataforma continental o zona económica exclusiva, según los artículos 17 y 18 del nuevo marco regulatorio.

Como condición para la distribución de beneficios se establece que los productos acabados tengan un componente del patrimonio genético o de CT asociado como elemento principal de valor agregado y consten en la Lista de Clasificación de Distribución de Beneficios que será publicada por la Unión.

Quedan exentas de la distribución de beneficios las operaciones de licencia, transferencia o permiso de uso de cualquier forma de derecho de propiedad intelectual sobre el producto acabado, procedimiento o material reproductivo; la exploración económica de producto acabado o de material reproductivo originario del acceso al patrimonio genético de especies introducidas que no formen populaciones espontáneas y que no hayan adquirido características distintivas propias del país; y también la exploración económica del producto intermedio y de procesos, así como la exploración económica de material reproductivo dentro del eslabón de la cadena productiva, conforme el artículo 17 de la mencionada ley en comento. Se enuncian también otras exenciones tales como microempresas, empresas de pequeño porte, micro emprendedores individuales, agricultores tradicionales y sus cooperativas, fa- 
bricantes de productos intermedios o desarrolladores de procesos, así como los productores de material reproductivo que comercialicen exclusivamente para otros productores dentro de la cadena productiva para fines de multiplicación de ese material.

El porcentaje de repartición de beneficios para el acceso al patrimonio genético varía entre 1 y $0,1 \%$ de los ingresos netos anuales obtenidos con la exploración económica del producto o material reproductivo. Con total acierto legal, la modalidad no monetaria de retribución incluye el proyecto para: la conservación o uso sostenible de la biodiversidad o para protección y manutención del conocimiento, innovación o prácticas de la población indígena, la comunidad tradicional o el agricultor tradicional; la transferencia de tecnología (y aquí en enlace oportuno con la figura de la Propiedad Intelectual); la disponibilidad en el dominio público (en este caso sin protección por derecho de PI o restricción tecnológica) del producto desarrollado a partir del acceso; la capacitación de recursos humanos en temas relacionados a la conservación y al uso sostenible del patrimonio genético o del CT asociado; y la distribución gratuita en programas de interés social del producto acabado o del material reproductivo desarrollado a partir del acceso.

Los valores de repartición de beneficios serán destinados a la población indígena, la comunidad local o el agricultor tradicional por el acceso al conocimiento de origen identificable. El acuerdo deber ser formalizado mediante firma del Término de Distribución de Beneficios Celebrado entre el proveedor y el usuario del conocimiento. Cuando se utilice el CT asociado de origen no identificable o difuso, todas las poblaciones indígenas, comunidades locales y agricultores tradicionales existentes en el país serán considerados beneficiarios, y recordemos que la ley prevé que cuando el acceso se refiere al CT asociado de origen no identificable o difuso, no existe la necesidad de consentimiento previo informado.

Los casos de incumplimiento de los procedimientos previstos, ya sea por acción u omisión del nuevo marco regulatorio, serán sancionados como infracción administrativa que varía desde la advertencia, multa, decomiso de muestras de patrimonio genético o de los productos derivados de tales muestras o del CT asociado, incluyendo la cancelación del registro y de la patente, si fuere el caso.

Ahora bien, la situación de la propiedad intelectual de los CT en México es un tema complicado porque no existe un cuerpo legal, y la normatividad vigente se encuentra dispersa en diferentes leyes afines que fueron detalladas anteriormente y que tienen mayormente que ver con lo que se denomina en este país como Derecho Ambiental. Esta ausencia de legislación para proteger el conocimiento tradicional es resultado de no considerar a los saberes, actividades y prácticas locales como un conocimiento formal. El argumento para no reconocer y proteger los conocimientos tradicionales es que constituyen patrimonio común o de dominio público. Ante esta situación, se precisa hacer estudios empíricos para documentar los conocimientos tradicionales, y determinar si en la gestión de éstos intervienen procesos para la generación de productos. ${ }^{9}$

La Constitución Nacional establece en su artículo 2 que "La Nación mexicana es 
única e indivisible”. La Nación tiene una composición pluricultural sustentada originalmente en sus pueblos indígenas que son aquellos que descienden de poblaciones que habitaban en el territorio actual del país al iniciarse la colonización y que conservan sus propias instituciones sociales, económicas, culturales y políticas, o parte de ellas. La conciencia de su identidad indígena deberá ser criterio fundamental para determinar a quiénes se aplican las disposiciones sobre pueblos indígenas. Son comunidades integrantes de un pueblo indígena, aquellas que formen una unidad social, económica y cultural, asentadas en un territorio y que reconocen autoridades propias de acuerdo con sus usos y costumbres. El derecho de los pueblos indígenas a la libre determinación se ejercerá en un marco constitucional de autonomía que asegure la unidad nacional. El reconocimiento de los pueblos y comunidades indígenas se hará en las constituciones y leyes de las entidades federativas, las que deberán tomar en cuenta, además de los principios generales establecidos en los párrafos anteriores de este artículo, criterios etnolingüísticos y de asentamiento físico. A) Esta Constitución reconoce y garantiza el derecho de los pueblos y las comunidades indígenas a la libre determinación y, en consecuencia, a la autonomía para: I. Decidir sus formas internas de convivencia y organización social, económica, política y cultural. II. Aplicar sus propios sistemas normativos en la regulación y solución de sus conflictos internos, sujetándose a los principios generales de esta Constitución, respetando las garantías individuales, los derechos humanos y, de manera relevante, la dignidad e integridad de las mujeres. La ley establecerá los casos y procedimientos de validación por los jueces o tribunales correspondientes. III. Elegir de acuerdo con sus normas, procedimientos y prácticas tradicionales, a las autoridades o representantes para el ejercicio de sus formas propias de gobierno interno, garantizando la participación de las mujeres en condiciones de equidad frente a los varones, en un marco que respete el pacto federal y la soberanía de los Estados. IV. Preservar y enriquecer sus lenguas, conocimientos y todos los elementos que constituyan su cultura e identidad. V. Conservar y mejorar el hábitat y preservar la integridad de sus tierras en los términos establecidos en esta Constitución. VI. Acceder, con respeto a las formas y modalidades de propiedad y tenencia de la tierra establecidas en esta Constitución y a las leyes de la materia, así como a los derechos adquiridos por terceros o por integrantes de la comunidad, al uso y disfrute preferente de los recursos naturales de los lugares que habitan y ocupan las comunidades, salvo aquellos que corresponden a las áreas estratégicas, en términos de esta Constitución. Para estos efectos las comunidades podrán asociarse en términos de ley. VII. Elegir, en los Municipios con población indígena, representantes ante los ayuntamientos. Las constituciones y leyes de las entidades federativas reconocerán y regularán estos derechos en los Municipios, con el propósito de fortalecer la participación y representación política de conformidad con sus tradiciones y normas internas. VIII. Acceder plenamente a la jurisdicción del Estado. Para garantizar ese derecho, en todos los juicios y procedimientos en que sean parte, individual o colectivamente, se deberán tomar en cuenta sus costumbres y especificidades culturales respetando los preceptos de esta Constitución. Los indígenas tienen en todo 
tiempo el derecho a ser asistidos por intérpretes y defensores que tengan conocimiento de su lengua y cultura. Las constituciones y leyes de las entidades federativas establecerán las características de libre determinación y autonomía que mejor expresen las situaciones y aspiraciones de los pueblos indígenas en cada entidad, así como las normas para el reconocimiento de las comunidades indígenas como entidades de interés público. B) La Federación, los Estados y los Municipios, para promover la igualdad de oportunidades de los indígenas y eliminar cualquier práctica discriminatoria, establecerán las instituciones y determinarán las políticas necesarias para garantizar la vigencia de los derechos de los indígenas y el desarrollo integral de sus pueblos y comunidades, las cuales deberán ser diseñadas y operadas conjuntamente con ellos. Para abatir las carencias y rezagos que afectan a los pueblos y comunidades indígenas, dichas autoridades, tienen la obligación de: I. Impulsar el desarrollo regional de las zonas indígenas con el propósito de fortalecer las economías locales y mejorar las condiciones de vida de sus pueblos, mediante acciones coordinadas entre los tres órdenes de gobierno, con la participación de las comunidades. Las autoridades municipales determinarán equitativamente las asignaciones presupuestales que las comunidades administrarán directamente para fines específicos. II. Garantizar e incrementar los niveles de escolaridad, favoreciendo la educación bilingüe e intercultural, la alfabetización, la conclusión de la educación básica, la capacitación productiva y la educación media superior y superior. Establecer un sistema de becas para los estudiantes indígenas en todos los niveles. Definir y desarrollar programas educativos de contenido regional que reconozcan la herencia cultural de sus pueblos, de acuerdo con las leyes de la materia y en consulta con las comunidades indígenas. Impulsar el respeto y conocimiento de las diversas culturas existentes en la nación. III. Asegurar el acceso efectivo a los servicios de salud mediante la ampliación de la cobertura del sistema nacional, aprovechando debidamente la medicina tradicional, así como apoyar la nutrición de los indígenas mediante programas de alimentación, en especial para la población infantil. IV. Mejorar las condiciones de las comunidades indígenas y de sus espacios para la convivencia y recreación, mediante acciones que faciliten el acceso al financiamiento público y privado para la construcción y mejoramiento de vivienda, así como ampliar la cobertura de los servicios sociales básicos. V. Propiciar la incorporación de las mujeres indígenas al desarrollo, mediante el apoyo a los proyectos productivos, la protección de su salud, el otorgamiento de estímulos para favorecer su educación y su participación en la toma de decisiones relacionadas con la vida comunitaria. VI. Extender la red de comunicaciones que permita la integración de las comunidades, mediante la construcción y ampliación de vías de comunicación y telecomunicación. Establecer condiciones para que los pueblos y las comunidades indígenas puedan adquirir, operar y administrar medios de comunicación, en los términos que las leyes de la materia determinen. VII. Apoyar las actividades productivas y el desarrollo sustentable de las comunidades indígenas mediante acciones que permitan alcanzar la suficiencia de sus ingresos económicos, la aplicación de estímulos para las inversiones públicas y privadas que propicien la creación de empleos, la in- 
corporación de tecnologías para incrementar su propia capacidad productiva, así como para asegurar el acceso equitativo a los sistemas de abasto y comercialización. VIII. Establecer políticas sociales para proteger a los migrantes de los pueblos indígenas, tanto en el territorio nacional como en el extranjero, mediante acciones para garantizar los derechos laborales de los jornaleros agrícolas; mejorar las condiciones de salud de las mujeres; apoyar con programas especiales de educación y nutrición a niños y jóvenes de familias migrantes; velar por el respeto de sus derechos humanos y promover la difusión de sus culturas. IX. Consultar a los pueblos indígenas en la elaboración del Plan Nacional de Desarrollo y de los estatales y municipales y, en su caso, incorporar las recomendaciones y propuestas que realicen. Para garantizar el cumplimiento de las obligaciones señaladas en este apartado, la Cámara de Diputados del Congreso de la Unión, las legislaturas de las entidades federativas y los ayuntamientos, en el ámbito de sus respectivas competencias, establecerán las partidas específicas destinadas al cumplimiento de estas obligaciones en los presupuestos de egresos que aprueben, así como las formas y procedimientos para que las comunidades participen en el ejercicio y vigilancia de las mismas. Sin perjuicio de los derechos aquí establecidos a favor de los indígenas, sus comunidades y pueblos, toda comunidad equiparable a aquéllos tendrá en lo conducente los mismos derechos tal y como lo establezca la ley.

Puede notarse ausencia absoluta de la figura en comento en el artículo constitucional y estamos ante la misma y tímida problemática que enfrentan actualmente los países para regular el CT, como se viene anticipando.

Entre las otras disposiciones mencionadas destacan: la LGEEPA que establece la garantía del derecho de los pueblos indígenas a la protección, preservación, uso y aprovechamiento sustentable de los recursos naturales y la biodiversidad como uno de los pilares que el gobierno debe velar a la hora de trazar y conducir políticas públicas en materia ambiental. Además, al hacer mención de las "áreas protegidas” anuncia la generación, rescate y divulgación de conocimientos, prácticas y tecnologías tradicionales que permitan la preservación y el aprovechamiento sustentable, entre otros, con miras a proteger la cultura y la identidad de los pueblos indígenas. Ahora bien, la relevancia del artículo 102 de la LGDFS está en que se prevé la declaración de nulidad de patentes relativas a recursos genéticos forestales que no reconozcan los derechos de comunidades indígenas a la propiedad, conocimiento y uso de las variedades locales, así como el reconocimiento de la coexistencia de derechos de aprovechamiento de vida silvestre en un mismo predio, por un lado, por parte de sus propietarios o legítimos poseedores (artículo 4 LGVS) y, por otro, de integrantes de comunidades rurales con fines de rituales y ceremoniales (artículo 93 LGVS), todo lo cual no obstante suele quedar en simple retórica y que se plasma en la falta de regímenes sui generis en la materia y en iniciativas frustradas de legislar el acceso y al distribución equitativa de beneficios (ARGÜELLO; RAMOS; CADENA, 2008; MORENO et al, 2008).

Ejemplificando nuevamente, una Recomendación de 2002 de la Comisión Nacional de Derechos Humanos de México solicitaba la modificación del Programa de Manejo de la Reserva 
de la Biósfera del Alto Golfo de California y Delta del Río Colorado, a fin de atender necesidades económicas y culturales de la comunidad Cucapá a fin de hacer valer los derechos de territorio en conjunto con derechos culturales y económico y, tangencialmente, los de Propiedad Intelectual.

\section{CONCLUSIÓN}

La sustentabilidad es una preocupación global. Varios documentos internacionales como internacionales enumerados en esta investigación intentan salvaguardar las condiciones de un ambiente saludable para el presente y futuro de las generaciones, subrayando el respecto y la defensa de los intereses de los pueblos indígenas y de las comunidades tradicionales.

Atenta y partícipe a la normativa internacional de la cual los países analizados -Brasil y México- forman parte a través de la pertinente adhesión, el intento, aunque tímido aún, procura adecuarse y regular el acceso a la biodiversidad y a los conocimientos tradicionales agregados. En el año 2001, surge como parteaguas en Brasil la normativa que disciplina la forma de acceso, la protección al conocimiento tradicional asociado, previendo la distribución de los beneficios logrados de la exploración económica del producto o del proceso desarrollado como resultado del mismo e inclusive, la transferencia de tecnología. Las exigencias del CGEN y las demás regulaciones, por un lado, restringían la exploración de la biodiversidad y, por otro, creaba trabas burocráticas complejas para el acceso al desarrollo de las investigaciones, tal como la necesidad de una autorización previa al inicio de la investigación acompañada de un conjunto de documentos. Como una forma de reglamentar el acceso a los conocimientos tradicionales surge la nueva Ley 13120 en el año 2015, la cual representa un avance sólido en el sentido de dotar de un marco legal y facilitar la actuación de investigadores y exploradores de productos de la biodiversidad, por medio de la reducción de la burocracia y en medio de un ambiente más favorable a tal investigación, reduciendo las limitaciones en el ejercicio del trabajo de los mismos, así como de las empresas. Otro punto interesante es que las empresas extranjeras podrán desarrollar actividades de investigación sobre la biodiversidad brasileña desde el momento que se asocien a Universidades locales.

De esta manera, sin descuidar el respeto a la cultura y el patrimonio de los pueblos tradicionales y la distribución de los resultados por los conocimientos accesados, se destaca la relevancia de un genuino marco legal para el acceso al patrimonio genético y a los conocimientos tradicionales, estableciendo la división de los resultados con las comunidades tradicionales y una protección para estimular la investigación y la innovación que conduzcan al desarrollo sostenible presente y futuro, así como al acceso inclusivo a los beneficios de las nuevas (bio) tecnologías.

En México, país aún carente de normativa específica dentro de las normas de Propiedad Intelectual, por considerar que el conocimiento tradicional no es susceptible de apropiación alguna, y que se caracteriza por tener aristas normativas dispersas pero no específicas en la materia, la expectativa reside en reglamentación a través de una ley que defina las dimen- 
siones de acceso, permitiendo evaluar se realmente una norma positiva aparejará impulso al desarrollo de nuevas investigaciones o si más bien tendrá un efecto contrario donde lo intereses económica se superpongan a los ricos y primigenios intereses sociales y culturales de la vasta e histórica comunidad azteca.

\title{
TRADITIONAL KNOWLEDGE: SIMILARITIES BETWEEN THE BRAZILIAN AND THE MEXICAN LEGAL INSTRUMENTS ON INTELLECTUAL PROPERTY
}

\begin{abstract}
Traditional knowledge is understood as information transmitted from generation to generation, typically through oral communication, shared by specific communities and generated in a context associated with the culture of the group. The context of such knowledge is hard to delimit before scientific knowledge. These implications leave space for questions on the appropriation of goods that are considered world heritage, even if symbolically, such as biodiversity, so as to transform them into market products. In this way, this paper aims at analyzing how the new Brazilian regulatory mark, Law n. 13.123/2015, foresees the sharing of benefits in the sale of products which are based on traditional knowledge. It is noticeable that the new law makes it easier to access traditional knowledge and Brazilian biodiversity, with a governmental indication of expansion in research and innovation and, on the other hand, with the disagreement of traditional communities, denouncing exploratory conducts and based on a mostly economic point of view.
\end{abstract}

Keywords: Traditional knowledge. Traditional medicine. Intellectual property. Biodiversity. Brazilian Law n. 13.123/2015.

\section{CONHECIMENTOS TRADICIONAIS: APROXIMAÇÕES ENTRE OS MARCOS REGULATÓRIOS SOBRE PROPRIEDADE INTELECTUAL NO BRASIL E NO MÉXICO}

\section{RESUMO}

Os conhecimentos tradicionais são concebidos como as informações transmitidas de geração a geração, de forma tipicamente oral, compartilhadas por comunidades específicas e geradas em um contexto associado com a cultura de grupo. Inserem-se em um contexto de difícil delimitação frente ao conhecimento científico. Essas implicações dão margem a indagações sobre a apropriação de bens considerados patrimônios da humanidade, ainda que de forma simbólica, tal como a biodiversidade, para transformá-los em produtos. De tal modo, o presente trabalho de pesquisa busca analisar a maneira com que o novo marco 
regulatório brasileiro, a Lei 13.123/2015, prevê a repartição de benefícios na comercialização de produtos que baseiam no conhecimento tradicional. Percebe-se que a nova legislação flexibiliza o acesso aos conhecimentos tradicionais e à biodiversidade brasileira, com um indicativo governamental de expansão da pesquisa e da inovação e, por outro lado, a inconformidade das comunidades tradicionais, indicando condutas de caráter exploratório e com cores tendenciosamente econômicas.

Palavras-chave: Conhecimentos tradicionais. Medicina tradicional. Propriedade intelectual. Biodiversidade. Lei brasileira 13.123/2015.

1 Brasil ha ratificado mucho más tarde: apenas en el año 2002.

2 Algunos ejemplos de espécies brasileñas que fueron patentadas en el exterior: se cita el caso más famoso que es el del profesor de la Facultad de Medicina de la ciudad de Riberão Preto, Sérgio Ferreira, que descubrió en el veneno de la cobra jararaca una sustancia capaz de controlar la presión arterial. Sin dinero para sus investigaciones, aceptó una colaboración con el laboratorio farmacéutico Bristol-Myers Squibb y, a cambio de recursos, la empresa registró la patente del principio activo Captopril, una marca que generaba ganancias al tiempo de esta investigación US\$2,5 millones al año y que Brasil también se obliga a pagar.

$[\ldots]$

Bubiri - Sus semillas se utilizan desde hace siglos por los indios wapixana, de Roraima, como anticonceptivo. El laboratorio canadiense Bolink patentó el principio activo y ha desarrollado investigación con la sustancia para tratar el SIDA.

$[\ldots]$

Curare - Mezcla de hierbas guardada com mucha cautela por los índios y utilizada en la punta de la flecha como veneno para inmovilizar a la presa. Fue patentada por los Estados Unidos en los años 40 y es utilizado para producir relajante y anestésico quirúrgico.

"Espinheira santa" - Su extracto es un excelente remedio contra la gastritis. La empresa japonesa Nippon Mek Japan patentó sus propiedades.

Jaborandi - Ya transformado en remedio (Salegen) por el laboratorio farmacéutico Merck, la planta es un antídoto contra la xerostoma (dificultad para salivar). Investigaciones hechas por el mismo laboratorio, con base en la cultura indígena y de los caboclos brasileños (mezcla de blanco con indio) están con vistas a producir un remedio contra la calvicie. El jaborandi también es bueno para combatir infecciones pulmonares.

$[\ldots]$

Rompe-piedra- Usada por los indios para tratar problemas hepáticos y renales, fue patentada por un laboratorio norteamericano para fabricar un medicamento que trata la Hepatitis B.

3 Convención sobre Diversidad Biológica, artículo 1.

4 Entiéndase "nación" como el sentimiento de pertenencia social que constriñe espontáneamente al ser humano a un determinado grupo social, más allá de la zona geográfica donde haya nacido.

5 La OMPI (2017, online) define la Propiedad Intelectual como la "[...] suma de los derechos relativos a las obras literarias, artísticas y científicas, a las interpretaciones de los artistas intérpretes y a las ejecuciones de los artistas ejecutantes, a los fonogramas y a las emisiones de radiodifusión, a las invenciones en todos los dominios de la actividad humana, a los descubrimientos científicos, a los dieños y modelos industriales, a las marcas industriales, comerciales y de servicio, así como a las firmas comerciales y denominaciones comerciales, a la protección contra la competencias desleal y todos los demás derechos inherentes a la actividad intelectual en los dominios industrial, científico, literario y artístico."

6 En Brasil, la Ley 9279, de fecha 14 de mayo 1996, disciplina estas materias.

7 Según Douglas Gabriel Domingues, hubo una decisión anterior de la Corte germánica involucrando el asunto. Sin embargo, ésta se refería al BGH, reportándose "las funciones biológicas del microorganismo per se como proceso o medio idóneo de producir un resultado final de un proceso mixto en que fueron empleados seres vivos y objetos inanimados." (DOMINGUES, 1989a, p. 7). 
8 Ley denominada de "Acceso y participación en los beneficios derivados de los recursos genéticos y los conocimientos tradicionales conexos" o Ley $\mathrm{N}^{\circ} 13123$ de fecha 20 de mayo de 2015. Tiene como Ley conexa el Decreto 8772 del 11 mayo de 2016, que la regula. La Ley 13123 deroga la Ley Provisional 2186-16 del 23 de agosto de 2001 denominada "Herencia genética y Conocimientos Tradicionales". Cfr. (BRASIL, 2015).

9 Ley denominada de "Acceso y participación en los beneficios derivados de los recursos genéticos y los conocimientos tradicionales conexos" o Ley $\mathrm{N}^{\circ} 13123$ de fecha 20 de mayo de 2015. Tiene como Ley conexa el Decreto 8772 del 11 mayo de 2016, que la regula. La Ley 13123 deroga la Ley Provisional 2186-16 del 23 de agosto de 2001 denominada "Herencia genética y Conocimientos Tradicionales". Cfr. (BRASIL, 2015).

\section{REFERÊNCIAS}

ADIERS, Cláudia Marins. A propriedade intelectual e a proteção da biodiversidade dos conhecimentos tradicionais. Revista da ABPI, Rio de Janeiro, n. 56, 2002.

ARGÜELLO, Rosa Josefina Bárcenas; RAMOS, Fernando Manzo; CADENA, Esther Méndez. La gestión del conocimiento tradicional y su relación con los derechos de propiedad intelectual. El caso del maíz criollo en una comunidad maya. Boletín Mexicano de Derecho Comparado, México, n. 123, 2008. Disponible en: < https://revistas.juridicas.unam. mx/index.php/derecho-comparado/article/view/4016/5123>. Consultado el: 17 abr. 2017.

BARBOSA, Denis Borges. Uma introdução à propriedade intelectual. Rio de Janeiro: Lúmen Júris, 1998.

BOFF, Salete Oro. Acordos e Tratados internacionais em matéria de patentes na biotecnologia e Legislação brasileira. In: DEL OLMO, Florisbal de Souza; ARAÚJO, Luís Ivani de Amorim (Coord.). O Direito de família contemporâneo e os novos direitos. Rio de Janeiro: Forense, 2006. p. 303-324.

BRASIL. Ley n ${ }^{\circ} 13.123$ de 20 de mayo de 2015. Reglamenta el inciso II del $\S 1$ y el $\S 4$ del art. 225 de la Constitución Federal, el artículo 1, el numeral J del artículo 8, el numeral C del artículo 10, el artículo 15 y los $\$ \$ 3$ y 4 del artículo 16 de la Convención sobre Diversidad Biológica, promulgada por Decreto 2.519, de 16 de marzo de 1998; dispone sobre el acceso al patrimonio genético, sobre la protección y el acceso al conocimiento tradicional asociado y sobre la distribución de beneficios para conservación y uso sostenible de la biodiversidad; revoca la Medida Provisoria 2.186-16, de 23 de agosto de 2001 y da otras providencias. Presidencia de la República, 2015. Disponible en: < https://www.planalto.gov.br>. Consultado el: 21 mayo 2015.

. Constitución (1988). Constitución (da) República Federativa del Brasil. Brasilia: Senado Federal, 1988. Disponible en: <http://www.planalto.gov.br>. Consultado el: 21 mayo 2015.

DOMINGUES, Douglas Gabriel. Privilégios de invenção, engenharia genética e biotecnologia. Río de Janeiro: Forense, 1989a. 
. Primeiras patentes de invenção de animal superior e a proteção legal dos embriões. Río de Janeiro: Forense, 1989b.

GORZ, André. O imaterial: conhecimento, valor e capital. Tradução Celso Azzan Júnior. San Pablo: Annablume, 2005.

INSTITUTO SOCIOAMBIENTAL. Disponible en: < https://www.socioambiental.org/pt-br>. Consultado el: 17 abril 2017.

MATTOS, Cláudio O. Justaposições como invento científico. Revista Biotecnologia, Ciência \& Desenvolvimento, Brasília, v. 1, n. 5, p. 26-27, mar./abr. 1998.

MÉXICO. Constitución Política de los Estados Unidos Mexicanos. Diario Oficial de la Federación, Ciudad de México, 5 feb. 1917. Disponible en: <http://www.ordenjuridico. gob.mx/Constitucion/cn16.pdf>. Consultado el: 17 abr. 2017.

. Ley General del Equilibrio Ecológico y la Protección al Ambiente. Diario Oficial de la Federación, Ciudad de México, 28 enero 1988. Disponible en: <http://biblioteca.semarnat.gob.mx/janium/Documentos/Ciga/agenda/DOFsr/148.pdf>. Consultada el: 18 abr. 2017.

. Ley General de Vida Silvestre. Diario Oficial de la Federación, Ciudad de México, 3 jul. 2000. Disponible en: < http://www.diputados.gob.mx/LeyesBiblio/pdf/146_191216.pdf >. Consultada el: 18 abr. 2017.

. Ley General de Desarrollo Forestal Sustentable. Diario Oficial de la Federación, Ciudad de México, 25 feb. 2003. Disponible en: <http://www.diputados.gob.mx/LeyesBiblio/ pdf/259_240117.pdf>. Consultada el: 18 abr. 2017.

. Ley General de Pesca y Acuacultura Sustentables. Diario Oficial de la Federación, Ciudad de México, 24 jul. 2007. Disponible en: <http://www.diputados.gob.mx/LeyesBiblio/ pdf/LGPAS_040615.pdf>. Consultada el: 18 abr. 2017.

. Ley de Propiedad Industrial. Diario Oficial de la Federación, Ciudad de México, 27 jun. 1991. Disponible en: <http://www.diputados.gob.mx/LeyesBiblio/pdf/50_010616. pdf $>$. Consultada el: 17 abr. 2017.

MIRANDA, Rafael Julio Pérez. Tratado de derecho de la propiedad industrial. 5. ed. México: Editorial Porrúa, 2011.

MIRANDA, Rafael Pérez; PICHARDO, Quetzalli de la Concha. Protección de los conocimientos tradicionales y de los vegetales en el Código Orgánico de la Economía Social de los Conocimientos de Ecuador. Revista Alegatos, México, n. 95, p. 27-44, mayo/agosto 2017.

MORENO, P. C. Breuner. Tratado de patentes de invencion. Buenos Aires: Abeledo-Perrot, 1957.v. 1.

MORENO, Rolando Cañas et al. Marco legal para el conocimiento tradicional sobre la biodiversidad. En: CAPITAL Natural de México: Conocimiento actual de la biodiversidad. 
México: Conabio, 2008. v. 1. Disponible en: <http://www.biodiversidad.gob.mx/pais/pdf/ CapNatMex/Vol\%20I/I17_Marcolegal.pdf>. Consultado el: 17 abr. 2017.

MORIN, Edgar. Ciência com consciência. Río de Janeiro: Bertrand Brasil, 1996.

OLIVEIRA, Ubirajara Mach de. A proteção jurídica das invenções de medicamentos e de gêneros alimentícios. Porto Alegre: Síntese, 2000.

ORGANIZACIÓN DE LAS NACIONES UNIDAS. Convenio sobre Diversidad Biológica. 1993. Disponible en: < https://www.cbd.int/doc/legal/cbd-es.pdf>. Consultado el: 18 abr. 2017.

ORGANIZACIÓN INTERNACIONAL DEL TRABAJO (OIT). Convenio 169 sobre Pueblos Indígenas y Tribales. 1989. Disponible en: < http://www.ilo.org/wcmsp5/groups/ public/@ed_norm/@normes/documents/publication/wcms_100910.pdf >. Consultado el: 18 abr. 2017.

.Convenio sobre Pueblos Indígenas y Tribales. 1989. Disponible en: <http://www.ilo. org/dyn/normlex/es/f?p=NORMLEXPUB:12100:0::NO::P12100_ILO_CODE:C169>. Consultado el: 18 abr. 2017.

ORGANIZACIÓN MUNDIAL DE LA PROPIEDAD INTELECTUAL (OMPI). Ley de Acceso y participación en los beneficios derivados de los recursos genéticos y los conocimientos tradicionales conexos. Disponible en: < http:/www.wipo.int/wipolex/es/text. jsp?file_id=376795>. Consultada el: 19 abr. 2017.

PAREJA, Enrique Láñez. Patentes biotecnológicas. Disponible en: <www.ugr.es/>. Consultado el: 24 jun 2002.

PÉREZ, Leyda Sughei Castillo. Régimen jurídico para la protección de la medicina tradicional y otros usos de la biodiversidad en las comunidades indígenas. México: Editorial Porrúa, 2006.

RIFKIN, Jeremy. O Século da biotecnologia. Tradução Arão Sapiro. San Pablo: MAKRON Books, 1999.

SHERWOOD, Robert M. Propriedade intelectual e desenvolvimento econômico. Tradução Heloísa de Arruda Villela. San Pablo: EDUSP, 1992.

SHIVA, Vendana. Biopirataria: a pilhagem da natureza e do conhecimento. Tradução Laura Cordellini Barbosa de Oliveira. Rio de Janeiro: Vozes, 2001.

WORLD HEALTH ORGANISATION (WHO). Traditional medicine. Disponible: <http://www.who.int/mediacentre/factsheets/fs134/en/>. Consultada el: 17 jun. 2010.

Submetido: 24 abr. 2017

Aprovado: 18 ago. 2017 